\title{
The Research on the 2D mobile game Platform Based on the Android System
}

\author{
Xiye Feng ${ }^{1, a}$, Meihui $\mathrm{Xu}^{2, \mathrm{~b}}$ \\ ${ }^{1}$ School of information Science \&Engineering, Qilu normal university, Jinan, 250013, China \\ ${ }^{2}$ School of information Science \&Engineering, University of Jinan, Jinan, 250022, China \\ aemail:Ikfxy@163.com, bemail:ise_xumh@ujn.edu.cn
}

Keywords: Android; game; Andengine; resource

\begin{abstract}
With the rapid development of smartphones, Android system by its unique openness, rich hardware selection, convenient application development, is accepted by more and more people. The mobile game based on Android, is gradually popular in the crowd. The game in the research with a beautiful picture and a wide variety of resources has the strong playability and a strong practical value. This platform is trying to use the Andengine to develop the game, combining the Android with Andengine engine which can let the game to run smoothly. The game with a business model provides a business interface for operators. This paper sets forth the corresponding algorithms used and it has the high theoretical and application value.
\end{abstract}

\section{Introduction}

With the development of science and technology, more and more people pay attention to mobile, portable and convenient, which makes the mobile game arises at the historic moment. As an open source operating system, Android mobile phone system is the first system for mobile terminals to build truly open and complete mobile applications. 2d game is one of those applications on the android platform. 2d mobile game is loved by people by its unique style, simple operation and smooth picture. However, the current mobile game market has many deficiencies. For example, the quality of the mobile game is coarse for the pursuit of short-term interests and low cost. Playability is much lower than traditional board game. Mobile game homogeneity is more and more serious and it is lack of innovation.

The game in the study is called SKY ADVENTURE, which is an adventure and agile game for android mobile phone users. The game picture is easy and lovely. Its pattern is novel and rich in content with outstanding technic. It includes the use of high-level programming languages such as Java, Android, Myeclipse development tools and AndEngine engine [1], combined with the database knowledge and operations.

The following is the simple introduction of the game.

(1) the name of the game

SKY ADVENTURE

(2) the game style

Style: Development of intelligence, adventure (AVG), role playing

(3) the operating environment

Operating system: android

Support environment: game platform for above Android system 2.2

\section{Function Framework}

This platform mainly involves in the four function modules such as Andengine engine module, interface module, data storage module, audio and video playback module. First we introduce the four modules simply. Figure 1 shows the main three function modules.

(1) Andengine engine module: It is responsible for the scene creation, initialization of characters and props and touch screen monitor about the game. It is the main part of the game. 
(2) The game interface module: It is responsible for the control interface implementation. Each interface is in the use of a separate layout file. It has a different buttons and ImageView.

(3) Data storage module: the game use SharedPreferences storage of data, which can create and modify and update the data.

(4) Audio and video playback modules: The video part is responsible for the opening of the animation playback of the game. The voice control module in the game is that we created a global variable which is used to control the game background music playback. We use a Boolean variable to record whether the play.

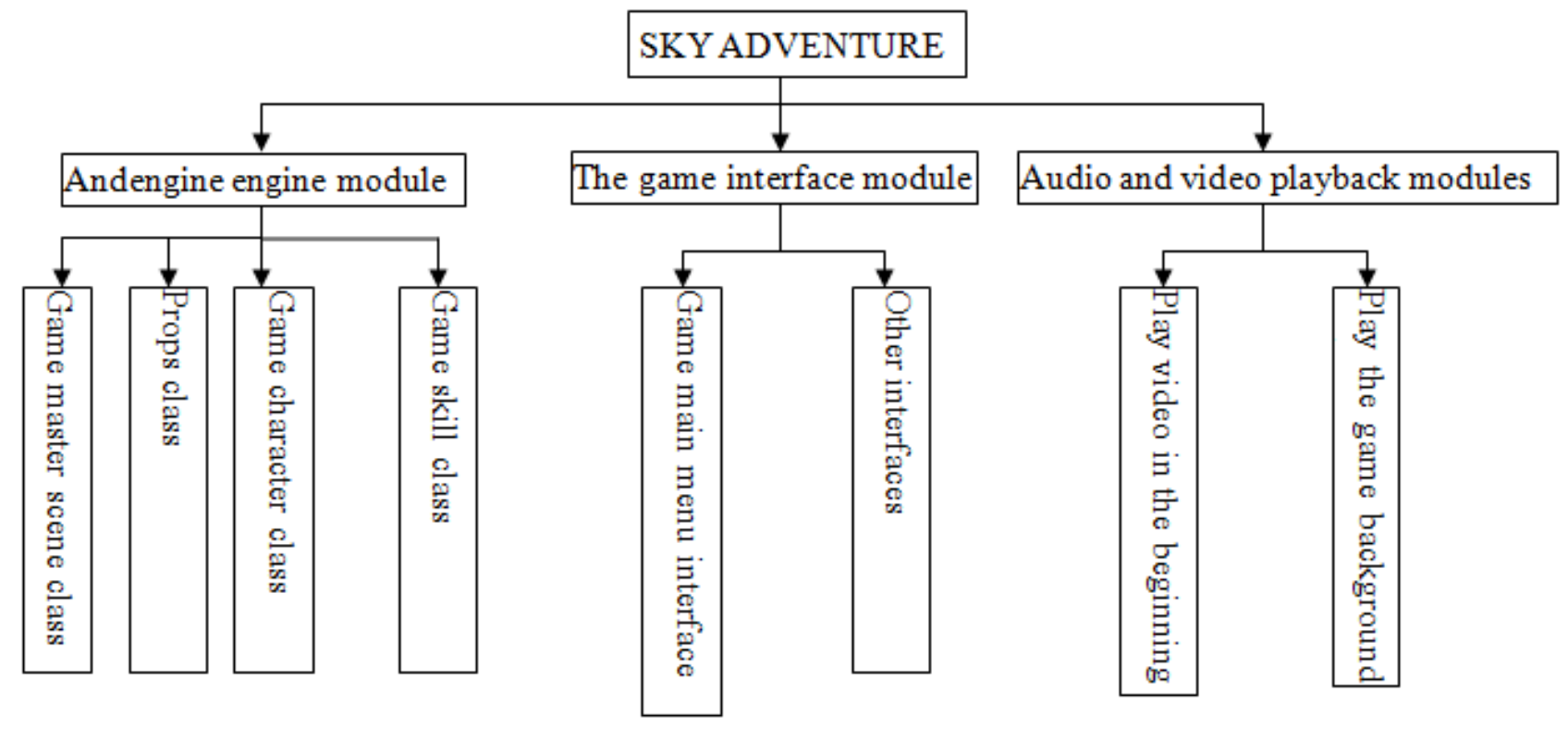

Fig.1. the functional framework of the platform

\section{Related Techniques and Algorithms Description}

The basic process. Figure 2 is the basic flow chart of the game.

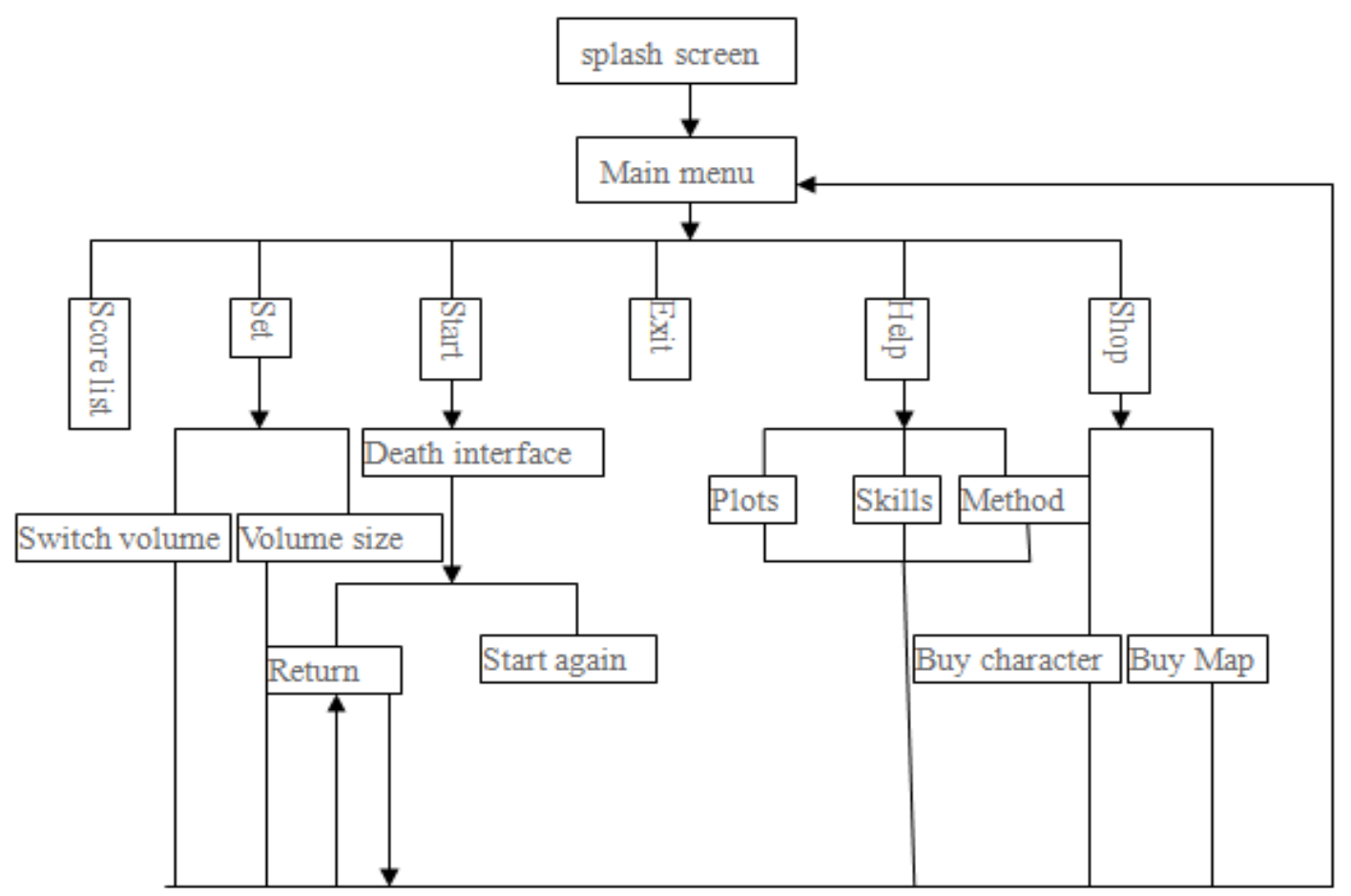

Fig.2. the basic flow chart of the game 
The related classes. Figure 3 is a related class diagram of the game.

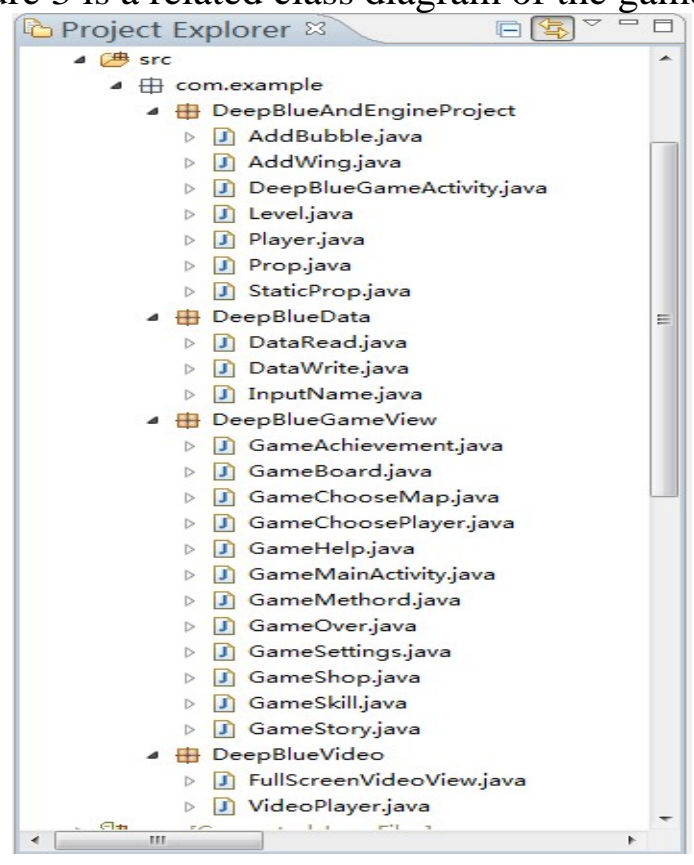

Fig.3. the related class diagram of the game

Figure 3 shows us there are four packages in the game. They are DeepBlueAndEngineProject, DeepBlueData, DeepBlueGameView etc. Each package has its own function. These packages [2] [3] have completed the scene layout design, character creation, data access, list of achievements and video playback.

Figure 4 shows the relation of the game modules [4].

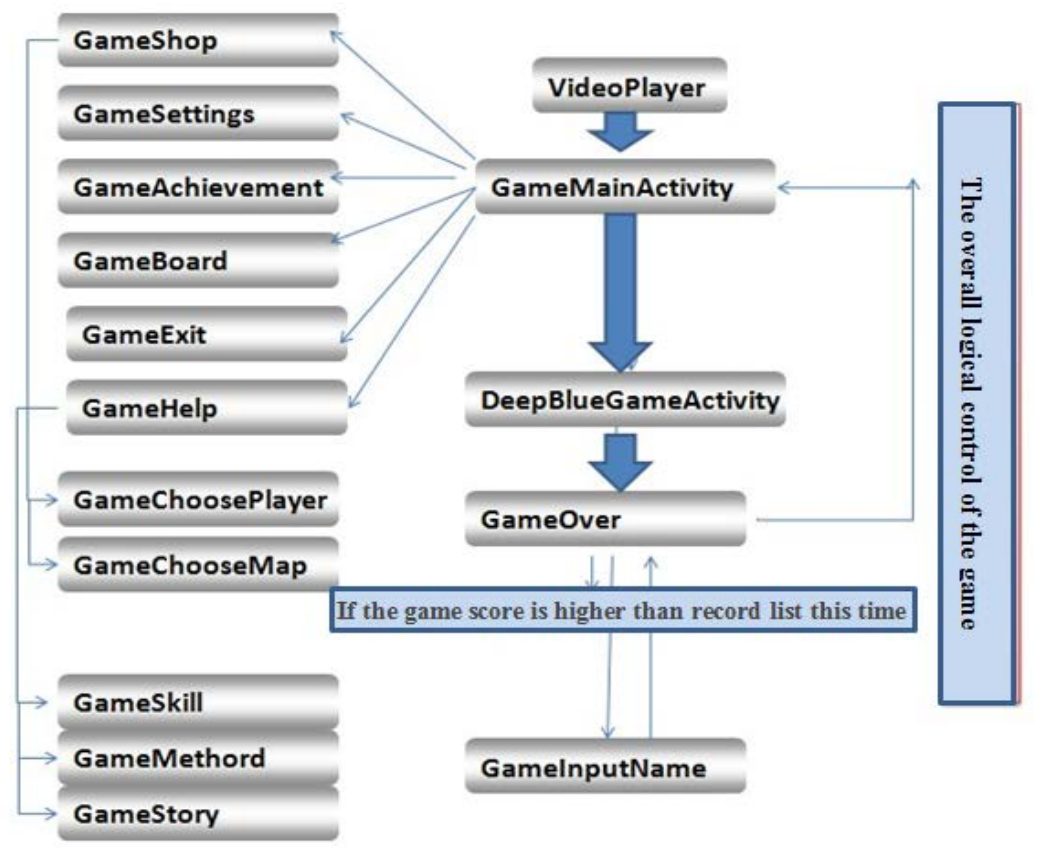

Fig.4. the relation of the game modules

Naming conventions in the program. Table 1 lists the components name in the program.

Table1. One of the components name tables

\begin{tabular}{|l|l|}
\hline \multicolumn{1}{|c|}{ components } & \multicolumn{1}{c|}{ abbreviations } \\
\hline EditText & txt \\
\hline Button & bth \\
\hline ListView & sp \\
\hline Spinner & lv \\
\hline
\end{tabular}


Results and achievements. Figure 5 is the main interface of the game platform. Click on the APP for the sky big adventure, we will enter the game main page. The home page includes the start, help, Settings, prominent, exit, the lower left corner shop and list of achievements etc.

This main story of the game is by these glittering energy tones which are in the lower left as shown in figure 6. Little monsters guided by energy stones, break through the sky, energy stone will accumulate in the game. Every 50 will be automatically reset, and then the corresponding character skill icon will move. Now the skills can be used by clicking on them.

There are two little monsters and two maps can be used for players in the game. Monsters blood is in the upper left corner of the interface. Next to the blood is the jumping height and it is used to calculate the final score. A pause button is in the top right corner, energy stone is in the lower left corner, the small monster skills is in the bottom right corner . Figure 5 shows them.

Algorithms Description. The algorithm for collision [5] and layout is more complex in the design of the game. Distance is commonly used in simple detection method to detect the collision test in the game platform.

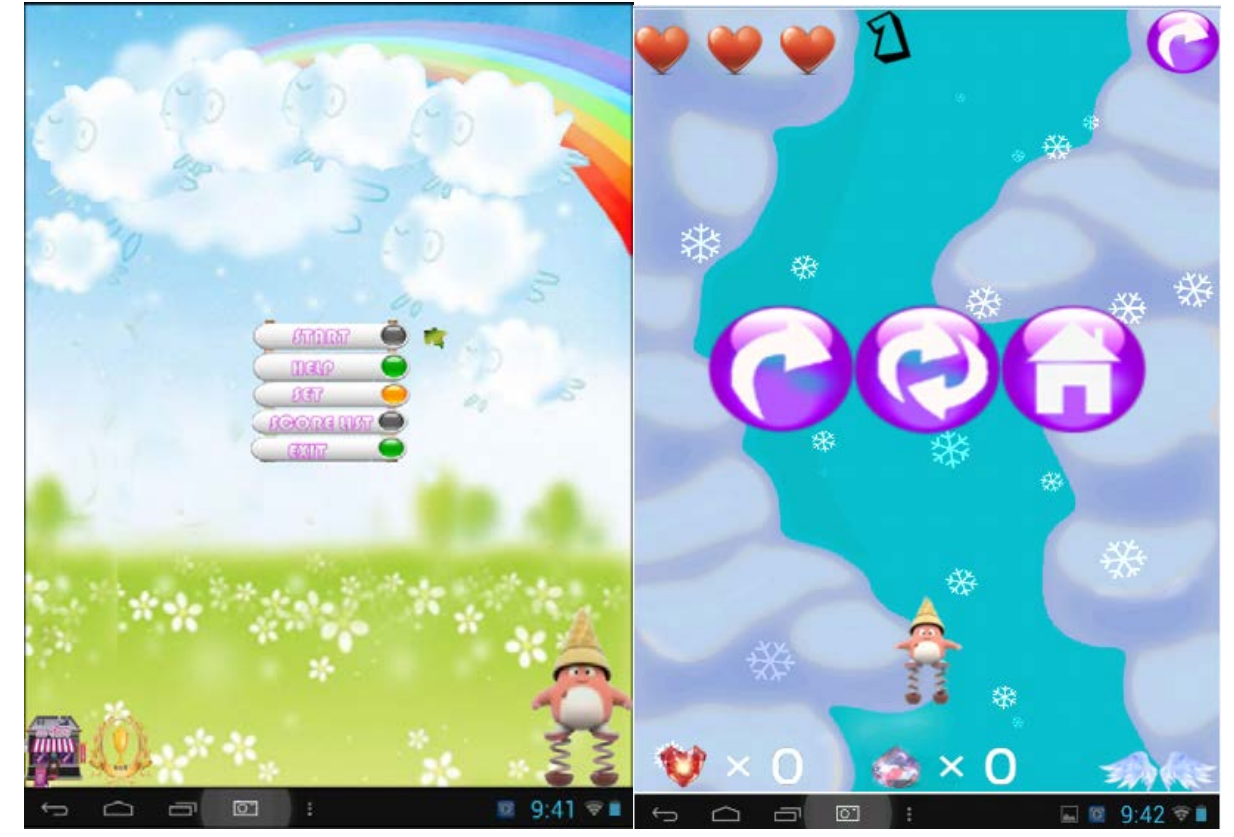

Fig.5. the main interface and one of the screenshots of the system

The principle of implementation is that by detecting whether two objects of rectangle are crossed.

We assume that the parameters of the rectangular $1:(\mathrm{x} 1, \mathrm{y} 1)$ are the coordinates of the upper-left corner, the width is $\mathrm{w} 1$, the height is h1. The parameters of the rectangular 2: (x2, y2) are the coordinates of the upper-left corner, the width is W2, the height is h2. During the collision of testing [6] [7], the parameters will satisfy the following mathematical expressions.

The $\mathrm{x}$ direction: $|(\mathrm{x} 1+\mathrm{w} 1 / 2)-(\mathrm{x} 2+\mathrm{w} 2 / 2)|<=|(\mathrm{w} 1+\mathrm{w} 2) / 2|$

The y direction: $|(\mathrm{y} 1+\mathrm{h} 1 / 2)-(\mathrm{y} 2+\mathrm{h} 2 / 2)|<=|(\mathrm{hl}+\mathrm{h} 2) / 2|$

This kind of collision detection algorithm is simple relatively. In the program, it is only needed to convert the above conditions to the code.

\section{Test results and conclusions}

Test. Each functional module of the system has been tested. The test can know whether the program is running normally. Test method is shown in table 2.

Main test module is: the physical simulation, logic control, data storage, collision detection, particle effects, achievement rank, music switch, image processing and game commercial system etc. Then we will give the introduction to the key test module.

1) The logic control. How to handle the correlations between various modules of the game is the first problem. A good game should have clear intuitive framework structure and convenient 
maintenance code. We test the touching and key events and drawing. We find that the algorithm can be running efficiently and orderly, avoiding infinite loop and beyond the scope of array.

2) Memory management. By optimizing algorithm, it can release timely needless resources to reduce unnecessary spending and the consumption of memory by the game.

3) Data store. It is need to save the records in this game and sort them. In addition, the game also needs to record some numerical further processing, so it is higher requirements for data storage.

4) Collision detection. Because the game is mainly running through the collision and bounce, so collision detection needs more details, including the impact Angle, strength etc. Detail disposal after the collision is needed to design attentively.

Table2. Test method

\begin{tabular}{|l|l|l|}
\hline \multicolumn{1}{|c|}{ State } & \multicolumn{1}{|c|}{ Running event } & \multicolumn{1}{c|}{ Corresponding action } \\
\hline Game running & $\begin{array}{l}\text { Press the home button in the } \\
\text { smartphone }\end{array}$ & Game pause and sound close \\
\hline Game running & $\begin{array}{l}\text { Press the lock screen button in } \\
\text { the smartphone }\end{array}$ & Music close \\
\hline Game running & Forced to exit the game & $\begin{array}{l}\text { Game over but the record is } \\
\text { not saved }\end{array}$ \\
\hline Game running & Operating system damaged & $\begin{array}{l}\text { Game over but the record is } \\
\text { saved }\end{array}$ \\
\hline
\end{tabular}

\section{Conclusions}

This game platform combines the Android with Andengine engine. The algorithm is optimized in the design of the game and greatly improves the operation efficiency. The game runs smoothly. The game with a business model can be used by the operators to provide business interface. Game is designed accomplished mode that allows players to play different experience. Reserved interface is developed in the game and it will then be developed rapidly once again in future according to the demand. It is possible from $2 \mathrm{~d}$ to $3 \mathrm{~d}$ mode and it has a good prospect.

\section{References}

[1] Jeffrey Jacobson, Michael Lewis. GAME ENGINES IN SCIENTIFIC RESEARCH, Communications of ACM. January 2002.

[2] Mark Dalrymple, Scott Knaster. Learn Objective-C on the Mac. Apress. 2009.

[3] Stephen C. Kochan. Programming in Objective-C 2.0. Addison-Wesley. 2009.

[4] Daniel Sanchez-Crespo Dalmau. Core Techniques and Algorithms in Game Programming. New Riders Publishing. 2003。

[5] T. H. Cormen, C. E. Leiserson, R. L. Rivest, C. Stein, "Introduction to Algorithms, 2ed,"[M] The MIT Press, 2001.

[6] Matthias Teschner,Bruno Heidelberger,Matthias Mtiller, Optimized spatial hashing for collision detection of deformable objects, Proceedings of Vision,Modeling,Visualization VMV'03,Germany Munich:VMV2003,2003,47-54.

[7] M. Tang, S. Curtis ,S. Yoon, Interactive continuous collision detection between deform able models using connectivity based culling[J], IEEE Transactions on Visualization and Computer Graphics,2009,15(4): 544-557. 